\title{
Possibility of a multibunch plasma afterburner for linear colliders
}

\author{
R. Maeda, ${ }^{1}$ T. Katsouleas, ${ }^{1}$ P. Muggli, ${ }^{1}$ C. Joshi, ${ }^{2}$ W. B. Mori, ${ }^{2}$ and W. Quillinan ${ }^{1}$ \\ ${ }^{1}$ University of Southern California, Los Angeles, California 90089-0484, USA \\ ${ }^{2}$ University of California at Los Angeles, Los Angeles, California 90024, USA \\ (Received 28 June 2004; published 8 November 2004)
}

\begin{abstract}
A concept for increasing the energy of a multibunch linear collider using plasma wakefields is examined. The realization of high beam quality and high efficiency (and high luminosity) requires more complexity than the original plasma afterburner concept proposed for doubling the energy of single bunch linear colliders. This paper discusses the possibilities of using alternate bunches in the train to drive the wake and accelerate upon it or alternately a few bunches to excite the wake and a single bunch to accelerate it. Simulation results indicate that an energy of collision/energy of linac ratio of 2.8 can be obtained with $4 \%$ energy spread and 0.29 relative luminosity by utilizing five drive bunches per accelerated bunch. The concept including transverse effects is modeled with 2D linear plasma wakefield theory.
\end{abstract}

DOI: $10.1103 /$ PhysRevSTAB.7.111301

PACS numbers: 41.75.Lx, 52.75.-d

\section{INTRODUCTION}

Recently a plasma afterburner concept was proposed for doubling the energy of a linear collider in which single electron and positron bunches collide at the interaction point of the collider [1]. In that concept, a part of each electron and positron bunch is used to drive large wakefields in relatively short plasma sections just before the collision point. The wakefields accelerate an appropriately phased second part of each bunch to higher energy. In many instances it is desirable to distribute the charge over multiple bunches rather than single colliding bunches (e.g., to reduce beam-beam and wakefield effects). The use of multiple bunches has been considered for raising the luminosity of future colliders such as the Next Linear Collider (NLC) and TESLA designs [2]. Here we consider the possibility of extending the afterburner concept to a future linear collider in which multiple rather than single bunches collide (i.e., in which a bunch train of electrons collides with all of the corresponding bunches in a counterpropagating train of positron bunches). If the spacing between the bunches is large compared to the time needed to refresh the plasma, then the single bunch afterburner concept can be applied in its original form, simply in a repetitive fashion. This was considered recently in Ref. [3].

In this paper we consider several ways in which the afterburner concept could be extended to take advantage of the multibunch structure of a future collider. The possibilities include using alternate bunches in the train to drive the wake and accelerate upon it or using alternately a few bunches to excite the wake and a single bunch to accelerate on it. Each of the variations involves tradeoffs between ease of implementation, beam quality (particularly energy spread), transformer ratio (maximum energy gain of the accelerated particles as a multiple of the incoming energy per particle), and the number of accelerated particles. The use of a train of bunches to drive a large plasma wake has been proposed previously by several authors [4] in the context of accelerating a single trailing bunch. Here we are considering a periodic train in which the accelerating and driving bunches are intermixed. Several scenarios are modeled with linear plasma wakefield theory and the trade-offs are discussed.

\section{THE MODEL PROBLEM}

We consider two colliding trains of bunches as illustrated in Fig. 1. The bunches enter plasma sections prior to collision and the wakefields in the plasma from some of the bunches are used to accelerate the other bunches. As a starting point we take the parameters for the bunch trains given in Table I. There are roughly $10^{8}$ particles in each microbunch corresponding to a peak current of roughly $1 \mathrm{kA}$. The plasma density is chosen to be in the range of $10^{14} \mathrm{~cm}^{-3}$ to be sufficient to support large wakefields. In the model calculations to follow, we choose to load one electron bunch per plasma wavelength. Within the limits of linear theory, this choice is flexible and any number of integer plasma wavelengths could be added to the spacing. In practice, the lifetime of the plasma wave is expected to be of the order of 10 periods [5]. Ten plasma periods between bunches would correspond roughly to one bunch per rf bucket at the $11.4 \mathrm{GHz}$ frequency of the NLC [3].

\section{PLASMA WAKEFIELD MODEL}

In contrast to the single bunch afterburner, multibunch collider designs have typically lower peak currents and as a result the plasma wakefields excited by the bunches are typically small enough to be modeled well with linear theory. The results we have obtained through linear theory are applicable to both positrons and electrons with the only difference being a sign change for positrons. Thus we analyze the multibunch plasma afterburner scenarios with the well-known Green's functions for the wakefield re- 


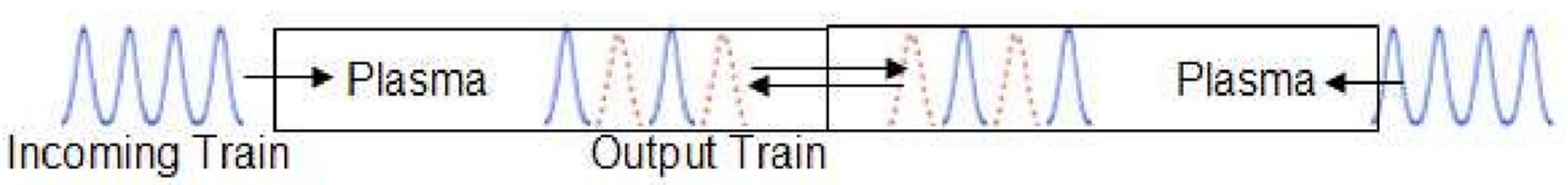

FIG. 1. (Color) Model problem. Wakefields in the plasma from some of the bunches are used to accelerate the others. The dotted line indicates the "spent" bunches. In this figure the even bunches (blue) in a four bunch train are accelerated by the wakes of the odd bunches (red).

TABLE I. Bunch train parameters.

\begin{tabular}{lc}
\hline \hline & $e^{-} / e^{+}$ \\
\hline$N_{m b}$ (particles in bunch) & $10^{8}$ \\
$N_{b}$ (number of bunches) & $>6$ \\
$\sigma_{z}$ (bunch length) & $75 \mu \mathrm{m}$ \\
$\sigma_{r}$ (beam radius) & $15 \mu \mathrm{m}$ \\
$C \tau$ (bunch spacing) & $3 \mathrm{~mm}$ \\
\hline \hline
\end{tabular}

sponse to an arbitrary relativistic bunch profile $n_{b}(\xi, r)$ [6]:

$$
\begin{gathered}
W_{Z}=Z^{\prime} R, \\
W_{r}=Z R^{\prime}, \\
Z=\left(\frac{4 \pi e}{k_{p}}\right) \int_{\infty}^{\xi} d \xi^{\prime} n_{b}\left(\xi^{\prime}\right) \sin k_{p}\left(\xi-\xi^{\prime}\right), \\
R(r)=\frac{k_{p}^{2}}{2 \pi} \int_{0}^{\infty} \int_{0}^{2 \pi} r^{\prime} d r^{\prime} d \theta^{\prime} f\left(r^{\prime}\right) K_{o}\left(k_{p}\left|\vec{r}-\vec{r}^{\prime}\right|\right),
\end{gathered}
$$

where $W_{Z}$ and $W_{r}$ are the longitudinal and transverse wake forces per unit charge, primes indicate derivatives with respect to ksi ( $\mathrm{ksi}$ is $z-c t$, the coordinate along the beam direction), and $n_{b}(\xi, r)=n_{b}(\xi) f(r)$.

\section{RESULTS}

The simplest scenario for a multibunch plasma afterburner is to choose the plasma wavelength (i.e., the plasma density) such that a half-integer multiple of the plasma wavelength fits between each microbunch. This is illustrated in Fig. 2 for a train of identical charge bunches. As we can see, the first bunch excites a wake that is completely absorbed by the second bunch and then the process repeats for bunches three and four, and so on. Ideally then, a train of $N$ bunches would enter each plasma and $N / 2$ bunches (the even ones) would emerge with twice the energy (per particle) of the initial bunch train. This corresponds to a transformer ratio of one, as can also be inferred from the ratio of the peak accelerating field on bunch two divided by the peak decelerating field on bunch one. A consequence of the total cancellation of the first bunch's wake by the second bunch is that the energy spread of the second bunch is large (the last particle experiences no field and gains zero energy). Used as an estimate for the energy spread, the range of fields experienced within the full width at half maximum of the beam length gives an energy spread of $\sim 100 \%$. Here we neglect both the phase slippage of the beam particles, since they are assumed ultrarelativistic, and radial variations of $E_{z}$ (because the beam is much smaller than the plasma skin depth $c / \omega_{p}$ ). We also neglect hosing or other instabilities of the beam or plasma inhomogeneities that could distort the shape of the beam or wake from that shown in Fig. 2.

For most high-energy physics applications, the energy spread in the example above is unacceptably large. To mitigate this as well as to increase the transformer ratio, we consider modifications to the bunch train. First we assume that we can make changes to the spacing between bunches; then we consider changing the charge in some of the microbunches. The first option may be difficult to realize experimentally. It may be possible to affect this change by chirping the bunch train at an earlier point in the accelerator and employing the dispersion in a chicane

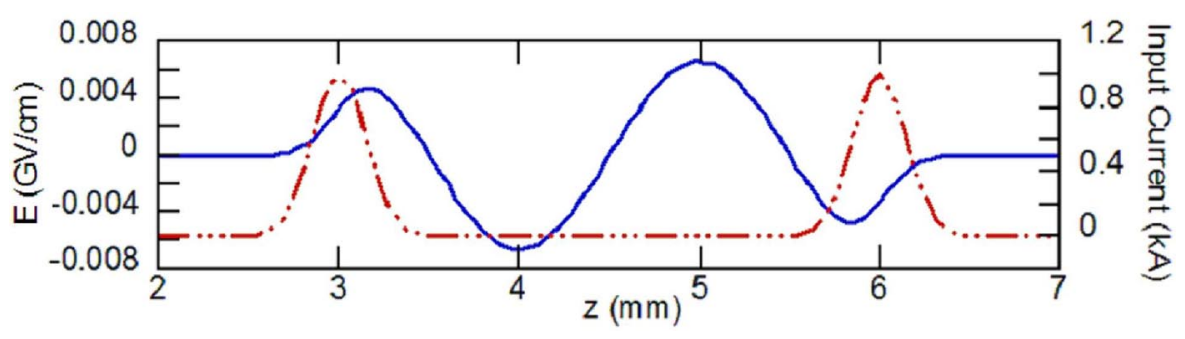

FIG. 2. (Color) Axial bunch current (dashed line) and resulting wakefield for identical bunches with plasma density such that a half-integer multiple of the plasma wavelength fits between each microbunch. 
magnet to slightly alter the spacing between bunches. Our goal here is not to consider a detailed realization of such a scheme but merely to point out the possibilities such changes would afford.

\section{A. Option 1: Identical bunches with varied spacing}

In order to achieve a small energy spread on the accelerated particles, we need to beam load only a portion of the wake. There are two ways to accomplish this: One is to make the accelerating wake larger by phasing a few bunches to drive the wake; the other is to decrease the number of particles in the accelerated bunch. These strategies have a further advantage in that they not only decrease energy spread but also increase the transformer ratio. Figure 3(a) shows a train of bunches in which two bunches are phased to drive the wake and the third is phased to absorb it and accelerate. (We choose the phase to approximately minimize the energy spread of the accelerated particles via the flattening of the wakefield by the accelerated bunches self-wake.) The process repeats with every other bunch accelerating. Since the wake absorption by the third bunch is not complete, there remains some wake energy to which the fourth bunch adds. This process is similar for all bunches except the first (because no wake energy precedes it). To compensate we have doubled the charge in the first bunch, all other bunches being the same. The larger wakefield within the first bunch, however, lowers the transformer ratio. To overcome this, we next divide the charge in the first bunch into several smaller bunches. Figures 3(b) and 3(c) illustrate using three and five initial drive bunches followed by a repeating pattern of two drive and one trailer bunch, respectively. For these two cases the process repeats with every third bunch accelerating.

From Fig. 3 we infer transformer ratios $(R=$ $E+/ E-)$ of $1-1.8$ and an energy spread of $4 \%-10 \%$, respectively (Table II). The ratio of the maximum collision energy to linac energy is given by $1+R$. We can further improve the transformer ratio and energy spread by employing more drive bunches per accelerated bunch. Of course, this reduces the number of accelerated particles and hence the luminosity (proportional to the square of the number of accelerated particles, $N_{\mathrm{acc}}^{2}$ ). Without invoking the extra focusing of plasma lenses [1], the luminosity of the scenarios in Figs. 2 and 3 would approach one-fourth $(1 / 2$ squared) and one-ninth $(1 / 3$ squared) of the initial bunch train luminosities, respectively.

\section{B. Option 2: Reduced bunch charge in alternate (load) bunches}

Figure 4(a) shows a train of bunches in which a single bunch drives the wake and the subsequent bunch, con-
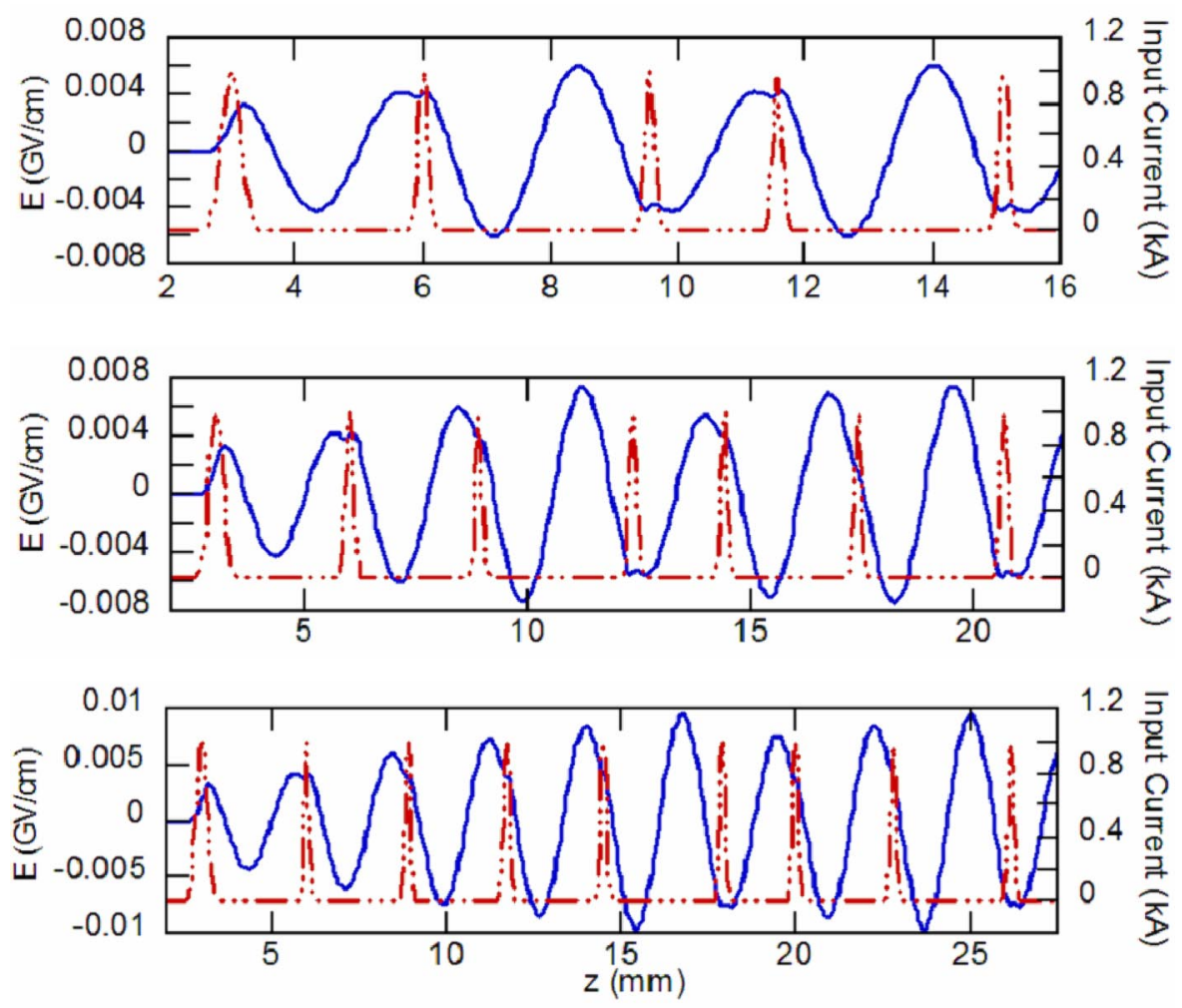

FIG. 3. (Color) Axial bunch current (dashed line) and longitudinal plasma wakefields for identical bunches with varied spacing: (a) two initial drive bunches, (b) three initial drive bunches, and (c) five initial drive bunches. 

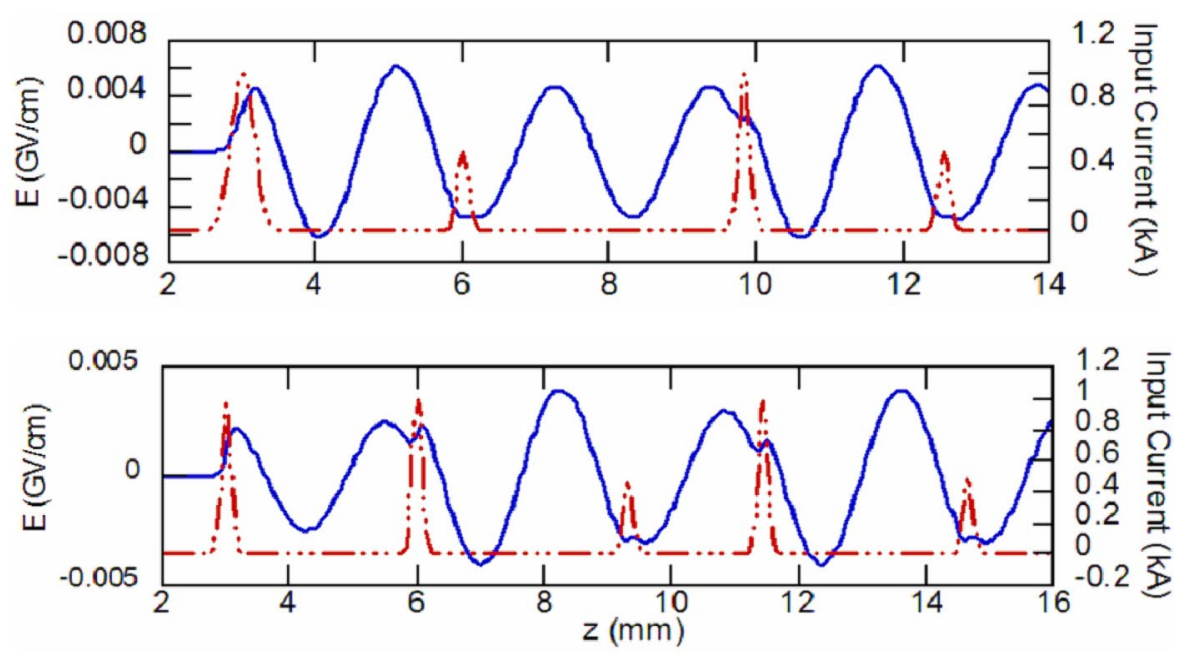

FIG. 4. (Color) Axial bunch current (dashed line) and longitudinal plasma wakefields for reduced bunch charge in alternate (load) bunches: (a) single initial drive bunch. Spacing between bunches is 3,3.84, and $2.71 \mathrm{~mm}$. Note that the pattern from $z=6$ to $12.5 \mathrm{~mm}$ repeats thereafter. (b) Two initial drive bunches. Spacing between bunches is $3,3.3,2.13$, and $3.21 \mathrm{~mm}$. Note that the pattern from $z=7$ to $12.5 \mathrm{~mm}$ repeats thereafter.

taining fewer particles than the driving bunch, is phased to absorb it and accelerate. Similar to option 1, we choose the phase to approximately minimize the energy spread of the accelerated particles. The process repeats with every other bunch being accelerated. Since the accelerated bunch contains fewer particles than the driving bunch, there remains wake energy to which the next driving bunch adds. To handle the issue of starting with no wake we used various approaches, including doubling the number of particles in the first bunch (in relation to the number of particles in all other driving bunches) as in Fig. 4(a) and using multiple drive bunches initially. Dividing the number of particles in the first bunch into two driving bunches containing the same number of particles lowers the retarding field and increases the transformer ratio. Figure 4(b) demonstrates using two initial driving bunches followed by a repeating pattern of alternating driving bunches and accelerated bunches containing half the number of particles. From Figs. 4(a) and $4(b)$ we infer transformer ratios $(E+/ E-)$ of $1-1.5$ and an energy spread of $2 \%-4 \%$, respectively. Table II summarizes the results for the various options modeled here. We comment that larger transformer ratios can be obtained by using even more drive bunches per accelerated bunch. However, as we see from Table II, increasing the transformer ratio and lowering the energy spread is accomplished at the expense of fewer accelerated particles.

\section{TRANSVERSE CONSIDERATIONS}

The plasma wakefields have both transverse and longitudinal components as described in Eqs. (1) and (2). The transverse wake can correspond to a large focusing or defocusing force on a microbunch, depending on its phase in the wake. To ensure that the beam is not defocused by the plasma we evaluate the transverse wakefield [Eq. (2)] for the cases described above. The corresponding results for Figs. 3 and 4 are shown in Figs. 5 and 6,

TABLE II. Energy of collision/energy of linac, energy spread, and luminosity at collider (relative to luminosity without the afterburner, assuming similar spot sizes and disruption). Last column assumes a 30 bunch train.

\begin{tabular}{lccc}
\hline \hline & Energy of collision/energy of linac & Energy spread & Relative luminosity at collider energy \\
\hline Identical equally spaced bunches & 2 & $100 \%$ & 0.5 \\
Option 1: varied spacing (2 driving, 1 accelerating) & & \\
Two initial drive bunches & 2 & $10 \%$ & $\sim 0.5$ \\
Three initial drive bunches & 2.5 & $6 \%$ & 0.29 \\
Five initial drive bunches & 2.8 & $4 \%$ & 0.29 \\
Option 2: varied charge (1 driving, 1 accelerating) & 2 & & \\
Single initial drive bunch & 2.5 & $4 \%$ & 0.32 \\
Two initial drive bunches & & $2 \%$ & 0.30 \\
\hline \hline
\end{tabular}



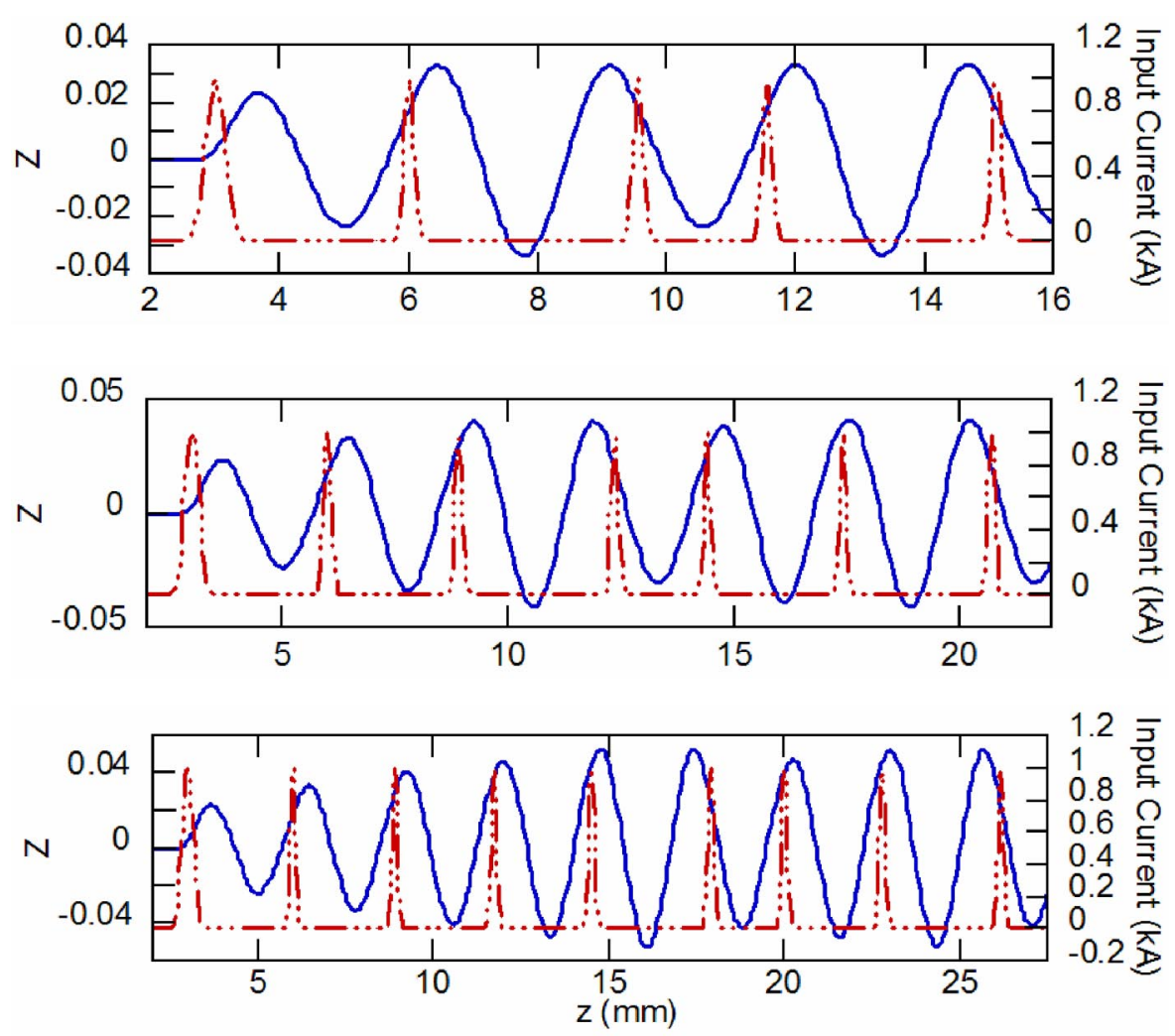

FIG. 5. (Color) Axial bunch current (dashed line) and transverse plasma wakefields [Eq. (3) in esu/cm] for identical bunches with varied spacing: (a) focusing forces for two initial drive bunches, (b) focusing forces for three initial drive bunches, and (c) focusing forces for five initial drive bunches.

respectively. For all the cases presented, all the bunches are in a phase to be focused. However, as can be seen in the figures, the focusing force varies along the bunch. This can lead to emittance growth if the beam is not matched [7] to this dynamic focusing field. The matched beam condition at a given emittance is for the spot size $\sigma$ to be

$$
\sigma=\left(\frac{m c^{2} \varepsilon_{N}^{2}}{\gamma e W_{r} / r}\right)^{1 / 4}
$$

where $\varepsilon_{N}$ is the normalized beam emittance and $\gamma$ is the beam's Lorentz factor.

Since the focusing force $W_{r}$ varies along the accelerated bunches' length, the matching condition (5) corre-
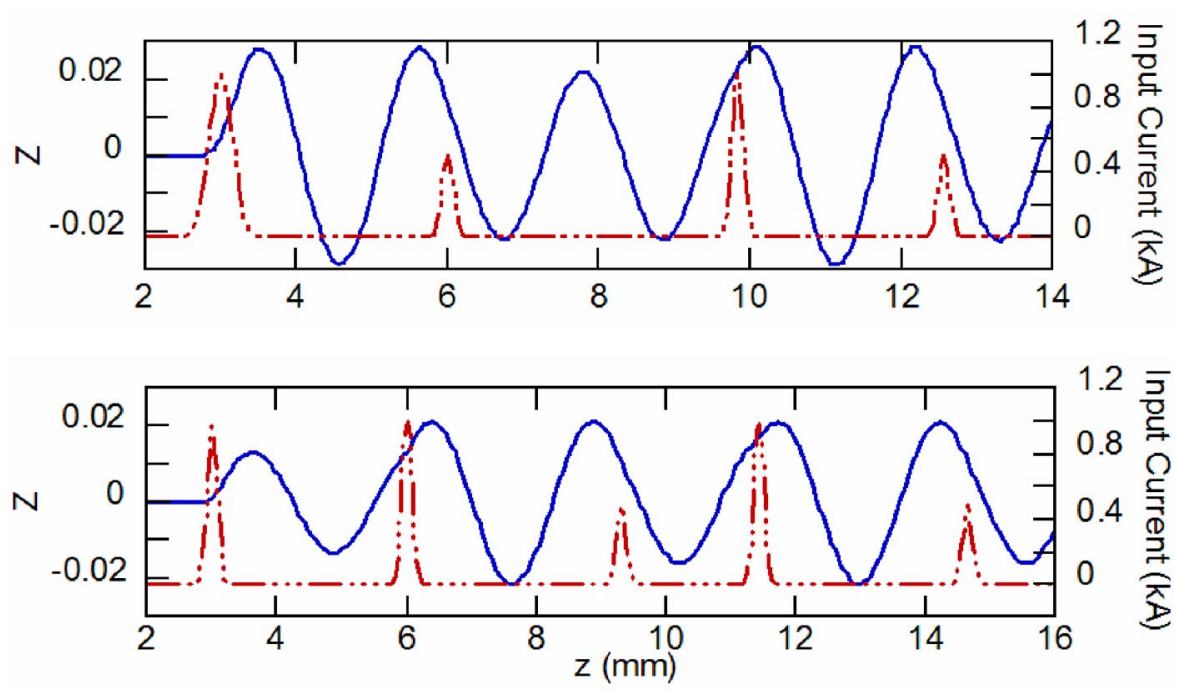

FIG. 6. (Color) Axial bunch current (dashed line) and transverse plasma wakefields for reduced bunch charge in alternate (load) bunches: (a) focusing forces for single initial drive bunch and (b) focusing forces for two initial drive bunches 
sponds to "ice cream cone" shaped beams. Previous particle simulations [8] of laser wakefield accelerators have shown that such a matching can be achieved via the use of a relatively short adiabatic plasma matching section in which the plasma wave amplitude ramps up (as a result of increasing laser intensity as the laser focuses). Further work is needed to see if a similar scheme could be applied here. Alternatively, hollow plasmas have been proposed as a means of eliminating transverse wakes entirely [9].

\section{DISCUSSION}

This work has shown the possibility of, as well as some of the issues associated with, extending the plasma afterburner concept to a multibunch collider. In general, such a realization is more complex than the original afterburner concept. We are led to consider customized bunch trains with varying spacing and charge per bunch. For example, using five drive bunches per accelerated bunch increases the energy at collision by a factor of up to 2.8 while maintaining an energy spread of $4 \%$ at the expense of 0.29 times the original luminosity. Some compensation for the luminosity reduction can be achieved with plasma lenses, but this is not considered in detail here. To achieve interesting accelerating gradients, shorter bunch spacing than is typical of current designs would be desirable. Alternatively, one could imagine using two linacs in the same accelerator, with one producing the drive train and the other producing the accelerated train. In this "two-beam accelerator" variant [10], it would be possible to make the drive and trail beams spacing quite small and hence to employ larger plasma densities and achieve larger accelerating gradients. Further work is needed both experimentally and with self-consistent simulations to address many of the questions raised by this work.

\section{ACKNOWLEDGMENTS}

The authors acknowledge Dave Sutter for discussions that led to this work and Robert Palmer and Ron Ruth for contributions to the idea. Preliminary simulations by M. Kazemi as part of a class project helped lead to this work. This work is supported by the NSF REU Program Grant No. PHY-0078715, NSF Grant No. PHY-0240909, and U.S. DOE Grant No. DE-FG02-92ER40745. The assistance of A. Ghalam and S. Deng is gratefully acknowledged.
TABLE III. Plasma densities used in figures.

\begin{tabular}{lcc}
\hline \hline Figure & $k_{p}\left(\mathrm{~mm}^{-1}\right)$ & Plasma density $\left(\mathrm{cm}^{-3}\right)$ \\
\hline 2 & 3.14 & $2.78 \times 10^{14}$ \\
3 and 5 & 2.36 & $1.56 \times 10^{14}$ \\
4(a) and 6(a) & 2.98 & $2.51 \times 10^{14}$ \\
4(b) and 6(b) & 2.51 & $1.78 \times 10^{14}$ \\
\hline \hline
\end{tabular}

\section{APPENDIX}

Table III lists the plasma densities used in the figures.

[1] S. Lee et al., Phys. Rev. ST Accel. Beams 5, 011001 (2002).

[2] T. Raubenheimer, www-project.slac.stanford.edu/lc/ local/notes/Video/121400TorNLC2000_config.pdf

[3] T. Raubenheimer, in Proceedings of the Advanced Accelerator Concepts Workshop (AAC'04), Stony Brook, NY, 2004 (AIP, to be published).

[4] A. Ogata, in Advanced Accelerator Concepts, edited by J. S. Wurtele, AIP Conf. Proc. No. 279 (AIP, New York, 1993), pp. 420-449; A. A. Bechtenev et al., in Advanced Accelerator Concepts, p. 466; A. K. Berezin et al., Fiz. Plazmy 20, 663 (1994); Plasma Phys. Rep. 20, 596 (1994); Plasma Phys. Rep. 20, 606 (1994), and references therein.

[5] J. R. Marquès, J. P. Geindre, F. Amiranoff, P. Audebert, J.C. Gauthier, A. Antonetti, and G. Grillon, Phys. Rev. Lett. 76, 3566 (1996); C.W. Siders, S. P. Le Blanc, D. Fisher, T. Tajima, M. C. Downer, A. Babine, A. Stepanov, and A. Sergeev, Phys. Rev. Lett. 76, 3570 (1996).

[6] T. Katsouleas, S. Wilks, P. Chen, J. M. Dawson, and J. J. Su, Part. Accel. 22, 81 (1987); R. Keinigs and M. E. Jones, Phys. Fluids 30, 252 (1987).

[7] S. Lee, T. Katsouleas, R. Hemker, and W. B. Mori, Phys. Rev. E 61, 7014 (2000); CE. Clayton, B. E. Blue, E. S. Dodd, C. Joshi, K. A. Marsh, W. B. Mori, S. Wang, P. Catravas, S. Chattopadhyay, E. Esarey, W. P. Leemans, R. Assmann, F. J. Decker, M. J. Hogan, R. Iverson, P. Raimondi, R. H. Siemann, D. Walz, T. Katsouleas, S. Lee, and P. Muggli, Phys. Rev. Lett. 88, 154801 (2002).

[8] W. Liu et al. (private communication).

[9] T.C. Chiou, T. Katsouleas, C. Decker, W. B. Mori, J. S. Wurtele, G. Shvets, and J. J. Su, Phys. Plasmas 2, 310 (1995).

[10] A. M. Sessler and S. S. Yu, Phys. Rev. Lett. 58, 2439 (1987). 